\title{
Min-Max-MSE Transceiver Design for MU-MIMO VLC System
}

\author{
Zhirong Zeng $\mathbb{D}^{1}{ }^{1}$ Huiqin Du $\mathbb{D}^{1},{ }^{1,2}$ and Zujian $W u \mathbb{D}^{3}$ \\ ${ }^{1}$ College of Information Science and Technology, Jinan University, Guangzhou, China \\ ${ }^{2}$ National Mobile Communications Research Laboratory, Southeast University, Nanjing, China \\ ${ }^{3}$ Jinan University-University of Birmingham Joint Institute at Jinan University (J-BJI), Guangzhou, China
}

Correspondence should be addressed to Huiqin Du; thuiqin.du@jnu.edu.cn

Received 3 February 2018; Revised 12 April 2018; Accepted 15 April 2018; Published 15 May 2018

Academic Editor: Imran S. Ansari

Copyright (C) 2018 Zhirong Zeng et al. This is an open access article distributed under the Creative Commons Attribution License, which permits unrestricted use, distribution, and reproduction in any medium, provided the original work is properly cited.

\begin{abstract}
This paper considers a multiuser (MU) multiple-input multiple-output (MIMO) visible light communication (VLC) system with broadcast channels. Due to the simultaneous transmission of the different source data to the receivers covered with the light rays, the interuser interference (IUI) may degrade the system performance. We strive to suppress the IUI by minimizing the maximum mean square errors (MSE) under the assumption of the perfect knowledge of channel state information (CSI). However, since the CSI may not be perfectly known in practice, a robust design is required against the channel uncertainties. Additionally, the nonnegativity and the limited linear range of the optical signals have been taken into account in the VLC transceiver designs. Simulation results validate that the proposed min-max-MSE approach can provide fair transmission, compared with the minimization of sum-MSE approach. Furthermore, it is demonstrated that the robust scheme is capable of providing robustness and gaining a considerable bit error rate (BER) performance.
\end{abstract}

\section{Introduction}

With the rapid development of the light-emitting diode (LED), a novel communication technology known as VLC gives rise to researchers' interests. Conventional bulbs are replaced by LEDs which can be used for both room illumination and high speed data communication $[1,2]$. Besides, visible light cannot penetrate walls because of its high frequency; as a result, indoor communications will not suffer from the intercell interference and security can be provided [3].

In an indoor environment, multiple lighting sources are often utilized for the whole room illumination and a higher transmission rate can be achieved through spatial multiplexing by constructing the MIMO VLC system [4-6]. Moreover, multiple users accessing the system simultaneously becomes a promising technique [7]. Due to users receiving all light rays from multiple lighting sources, the IUI may exist, which degrades the system performance. The IUI cancellation methods have been extensively investigated in MU radio frequency (RF) systems. Nevertheless, they cannot be applied directly to VLC systems because of the following reasons. (a) The VLC system adopts the intensity modulation and direct detection (IM/DD) scheme where the transmitted signals should be unipolar and nonnegative real values rather than bipolar and complex signals in RF systems.

(b) Owing to the VLC specific feature of providing stable brightness and meanwhile conveying information, the optical signal intensity is more limited with norm 1 constraint instead of the electronic power constraint in RF communication.

As a result, the above properties must be taken into account in the research of VLC systems. Many efforts are proposed to suppress the IUI for MU-MIMO VLC applications, such as sum-rate maximization [8-11], sum-MSE minimization [12], and max-MSE minimization [13]. However, the sum-rate maximization approach or the sum-MSE minimization method may introduce unfair transmission because the total sum rate and the total MSE are optimized. To consider the fairness among the multiple users, the minimization of the maximum MSE algorithm is adopted over the multiple-input single-output (MISO) VLC system [13] under the assumption of the perfect CSI. However, the CSI may not always be perfectly known and the channel uncertainties could lead to severe performance degradation when the transceiver design is only based on the estimated 


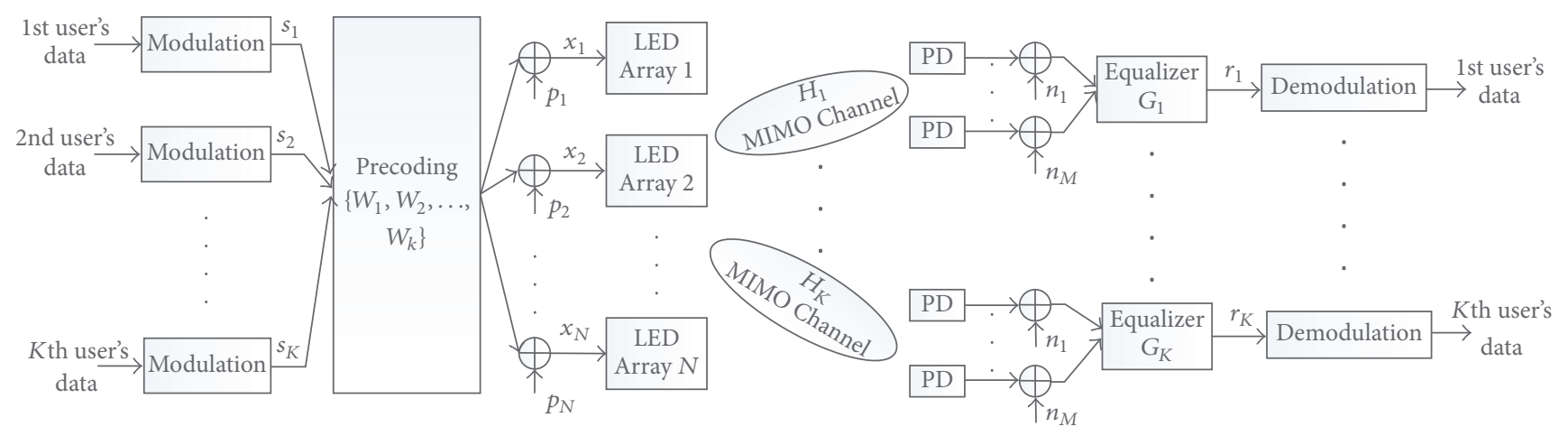

FIGURE 1: Schematic model of the proposed MU-MIMO VLC system.

channel information [14]. Therefore, it is critical to consider the channel imperfection in the VLC transceiver design.

In this work, we consider the indoor MU-MIMO VLC system and strive to eliminate the IUI by minimizing the maximum MSE. Due to the nonconvexity of the underlying problem with respect to multiple variables, the alternating minimization approach over variables is proposed to achieve the optimal solutions. The main contributions of this paper can be summarized as follows.

(1) Considering that the output signal of LED must be nonnegative and limited within the linear dynamic range, we impose the optical power constraint in the proposed design and the direct current (DC) bias is applied to provide stable brightness.

(2) Our proposed transceiver design optimizes the total MSE while providing throughput fairness for all users. The simulation and comparison results confirm the effectiveness of the proposed algorithm for guaranteeing the fairness with respect to the MSE performance.

(3) Besides the perfect CSI case, the CSI with uncertainties is also considered in our work, and the robust design is developed. The numerical results demonstrate that the robust scheme outperforms the nonrobust scheme and achieves considerable BER performance under the channel imperfection.

(4) Finally, we also investigate the impact of different geometric metrics, including users' relative locations, heights, and angles. The results imply that channel correlation is the most decisive factor for the system performance.

The rest of the paper is organized as follows. Section 2 presents the indoor MU-MIMO VLC system model. Section 3 investigates the optimal transceiver with the perfect channel information, and the robust scheme with imperfect CSI is developed in Section 4. The simulation results are discussed in Section 5 to verify the performance of the proposed iterative algorithm. Finally, the conclusions are drawn in Section 6.

Notation 1. In this paper, uppercase and lowercase letters denote matrices and vectors, respectively. The operators $\operatorname{vec}(\cdot),(\cdot)^{T}$, and $(\cdot)^{-1}$ stand for the vectorization, transpose, and inverse of a matrix or a vector, respectively. $|\cdot|$ represents the absolute operator, $\|\cdot\|_{1}$ denotes the $L_{1}$ norm of vectors, and $\|\cdot\|_{F}$ is the Frobenius norm. $\mathbb{E}_{\mathbf{X}}\{\cdot\}$ denotes the statistical expectation over $\mathbf{X} \cdot \operatorname{tr}(\cdot)$ is the trace of matrix. I is the identity matrix with corresponding dimensions. $(\cdot)^{1 / 2}$ represents the square root of a square matrix. The operator $\otimes$ denotes the Kronecker product.

\section{System Model}

The indoor VLC system consisting of $N$ transmitting LED arrays and $K$ user terminals equipped with multiple LEDs and multiple photodetectors (PD), respectively, is considered. Figure 1 illustrates the schematic model of the proposed VLC system, where the On-Off Keying (OOK) modulation is applied. LED arrays cooperate to transmit all data to multiple users through the light channels, and users can detect all the light rays leading to the IUI that may cause severe system performance degradation. Consequently, it is necessary to suppress the interference.

2.1. Transmitter Model. The white LED, which has the Lambertian radiation pattern, is chosen as the lighting source for the indoor VLC application. The radiant intensity can be expressed as

$$
R_{o}(\phi)=\frac{(m+1)}{2 \pi} \cos ^{m}(\phi),
$$

where $m=\ln 2 / \ln \left(\cos \Phi_{1 / 2}\right)$ is the order of Lambertian emission determined by the semiangle for half illuminance of the $\operatorname{LED} \Phi_{1 / 2}$ and $\phi$ is the angle of emission relative to the optical axis of the LED array.

The LED output signals can be written as

$$
\mathbf{x}=\sum_{k=1}^{K} \mathbf{W}_{k} \mathbf{s}_{k}+\mathbf{p},
$$

where the original signal for $k$ th user $\mathbf{s}_{k} \in \mathbb{R}^{l \times 1}$ is independent and identically distributed (i.i.d.) and bounded in $[-1,1]$ with the covariance matrix $\mathbf{R}_{\mathbf{s}_{k}}=\mathbb{E}\left\{\mathbf{s}_{k} \mathbf{s}_{k}^{T}\right\}=\sigma_{s}^{2} \mathbf{I} . \sigma_{s}^{2}$ is the variance of the input signal and $l$ represents the length of data stream. $\mathbf{W}_{k} \in \mathbb{R}^{N \times l}$ denotes the transmitter precoder and the DC bias vector $\mathbf{p}=\left[p_{1}, \ldots, p_{n}, \ldots, p_{N}\right]^{T} \in \mathbb{R}^{N \times 1}$ is added to satisfy the nonnegativity and linear-range requirement of LED input signals. 
Let $p_{\min }$ and $p_{\max }$ be the lower and upper bound of LED linear region; the optical power constraints for transmitter signals can be written as

$$
\begin{aligned}
& p_{n}-\sum_{k=1}^{K}\left|\mathbf{w}_{n k}\right| \geqslant p_{\min }, \\
& \sum_{k=1}^{K}\left|\mathbf{w}_{n k}\right|+p_{n} \leqslant p_{\max },
\end{aligned}
$$

$$
n=1, \ldots, N,
$$

where $\mathbf{w}_{n k} \in \mathbb{R}^{1 \times l}$ denotes the $n$th row vector for $k$ th precoding matrix $\mathbf{W}_{k}$.

As a result, the optical power constraint imposed on transmitting precoder can be rewritten as

$$
\sum_{k=1}^{K}\left|\mathbf{w}_{n k}\right|=\sum_{k=1}^{K}\left\|\mathbf{W}_{k}^{T} \mathbf{e}_{n}\right\|_{1} \leqslant p_{t}, \quad n=1, \ldots, N
$$

where $p_{t}=\min \left\{p_{n}-p_{\min }, p_{\max }-p_{n}\right\}$ and $\mathbf{e}_{n}$ denotes a zero vector with $n$th element being one; that is, $\mathbf{e}_{n}=$ $\left[\begin{array}{llll}\mathbf{0}_{1 \times(n-1)} & 1 & \mathbf{0}_{1 \times(N-n)}\end{array}\right]^{T}$.

2.2. VLC Channel Model. Several well-known channel models are used in the optical wireless transmission, such as the lognormal model $[15,16]$ and the indoor practical optical channel model $[6,17,18]$. The lognormal model is used to model free space optical (FSO) channel. In this work, the indoor practical optical channel model is adopted.

Assuming that there are no walls in the research model (e.g., an inner part of a larger indoor environment) and no barriers between transmitters and receivers, the reflections from walls can be ignored and only the light-of-sight (LOS) links are considered. Therefore, the VLC channel matrix model can be described as

$$
\mathbf{H}_{k}=\left[\begin{array}{cccc}
h_{11}^{k} & h_{12}^{k} & \ldots & h_{1 N}^{k} \\
h_{21}^{k} & h_{22}^{k} & \ldots & h_{2 N}^{k} \\
\vdots & \vdots & \ddots & \vdots \\
h_{M 1}^{k} & h_{M 2}^{k} & \ldots & h_{M N}^{k}
\end{array}\right],
$$

where each element $h_{m n}^{k}$ of channel matrix $\mathbf{H}_{k} \in \mathbb{R}^{M \times N}$ denotes the DC gain between $m$ th PD of the $k$ th user and $n$th LED array. It can be presented as

$$
\begin{aligned}
& h_{m n}^{k} \\
& = \begin{cases}\sum \frac{A R_{o}\left(\phi_{m n}\right)}{d_{m n}^{2}} g\left(\psi_{m n}\right) \cos \left(\psi_{m n}\right), & 0 \leqslant \psi_{m n} \leqslant \Psi, \\
0, & \psi_{m n}>\Psi,\end{cases}
\end{aligned}
$$

where $A$ is the detector area of the receiver and $d_{m n}$ is the distance between the central point of the $n$th LED array and $m$ th PD. $\psi_{m n}$ denotes the incidence angle relative to PD and
$\Psi$ represents the optical field of view (FOV) of PD. The gain of optical concentrator $g\left(\psi_{m n}\right)$ is defined as

$$
g\left(\psi_{m n}\right)= \begin{cases}\frac{\kappa^{2}}{\sin ^{2}(\Psi)}, & 0 \leqslant \psi_{m n} \leqslant \Psi, \\ 0, & \psi_{m n}>\Psi\end{cases}
$$

with the refractive index of concentrator $\kappa$.

For convenience of analysis, the arrayed LED transmitter is simplified as a point lighting source having the same power with the actual setting, where the LED spacing is fixed as $1 \mathrm{~cm}$ for simulations. Moreover, it is validated that, even in the limit case with $24 \mathrm{~cm}$ LED spacing, the maximum deviation of optical path loss is still acceptable [19]. Therefore, the simplification of arrayed LED transmitters for VLC channel modeling in this work is reasonable.

2.3. Receiver Model. At the $k$ th user terminal, the received signal vector can be written as

$$
\mathbf{y}_{k}=\mathbf{H}_{k} \mathbf{x}+\mathbf{n}_{k}=\mathbf{H}_{k} \mathbf{W}_{k} \mathbf{s}_{k}+\mathbf{H}_{k} \sum_{j \neq k} \mathbf{W}_{j} \mathbf{s}_{j}+\mathbf{H}_{k} \mathbf{p}+\mathbf{n}_{k},
$$

where $\mathbf{n}_{k} \in \mathbb{R}^{M \times 1}$ is the additive white Gaussian noise with zero mean and covariance matrix $\mathbf{R}_{\mathbf{n}_{k}}=\mathbb{E}\left\{\mathbf{n}_{k} \mathbf{n}_{k}^{T}\right\}=\sigma_{n}^{2} \mathbf{I}$. $\sigma_{n}^{2}$ is the receiver noise variance. The second term denotes the IUI which involves the interference signal $s_{j}(j \neq k)$. The corresponding received data of the $k$ th user can be presented as

$$
\mathbf{r}_{k}=\mathbf{G}_{k} \mathbf{y}_{k}=\mathbf{G}_{k} \mathbf{H}_{k} \sum_{j=1}^{K} \mathbf{W}_{j} \mathbf{s}_{j}+\mathbf{G}_{k} \mathbf{H}_{k} \mathbf{p}+\mathbf{G}_{k} \mathbf{n}_{k}
$$

where $\mathbf{G}_{k} \in \mathbb{R}^{l \times M}$ is defined as the linear equalizer.

\section{Optimal Transceiver Design with Perfect Channel State Information}

In order to recover the original source information and subtract the influence of the IUI efficiently, the precoder and equalizer are jointly optimized based on MMSE criteria. The corresponding MSE of the $k$ th user can be written as

$$
\begin{aligned}
\operatorname{MSE}_{k} & \left(\mathbf{r}_{k}, \mathbf{s}_{k},\left\{\mathbf{W}_{j}\right\}_{j=1, \ldots, k, \ldots, K}, \mathbf{G}_{k}\right) \\
= & \mathbb{E}_{\mathbf{s}_{k}, \mathbf{n}_{k}}\left\{\left\|\mathbf{r}_{k}-\mathbf{G}_{k} \mathbf{H}_{k} \mathbf{p}-\mathbf{s}_{k}\right\|_{\mathrm{F}}^{2}\right\} \\
= & \operatorname{tr}\left(\left(\mathbf{G}_{k} \mathbf{H}_{k} \mathbf{W}_{k}-\mathbf{I}\right) \mathbf{R}_{\mathbf{s}_{k}}\left(\mathbf{G}_{k} \mathbf{H}_{k} \mathbf{W}_{k}-\mathbf{I}\right)^{T}\right) \\
& +\sum_{j \neq k} \operatorname{tr}\left(\mathbf{G}_{k} \mathbf{H}_{k} \mathbf{W}_{j} \mathbf{R}_{\mathbf{s}_{j}}\left(\mathbf{G}_{k} \mathbf{H}_{k} \mathbf{W}_{j}\right)^{T}\right) \\
& +\operatorname{tr}\left(\mathbf{G}_{k} \mathbf{R}_{\mathbf{n}_{k}} \mathbf{G}_{k}^{T}\right)
\end{aligned}
$$




$$
\begin{aligned}
= & \sum_{j=1}^{K} \operatorname{tr}\left(\mathbf{G}_{k} \mathbf{H}_{k} \mathbf{W}_{j} \mathbf{R}_{\mathbf{s}_{j}}\left(\mathbf{G}_{k} \mathbf{H}_{k} \mathbf{W}_{j}\right)^{T}\right)+\operatorname{tr}\left(\mathbf{R}_{\mathbf{s}_{k}}\right) \\
& -\operatorname{tr}\left(\mathbf{G}_{k} \mathbf{H}_{k} \mathbf{W}_{k} \mathbf{R}_{\mathbf{s}_{k}}\right)-\operatorname{tr}\left(\mathbf{R}_{\mathbf{s}_{k}}\left(\mathbf{G}_{k} \mathbf{H}_{k} \mathbf{W}_{k}\right)^{T}\right) \\
& +\operatorname{tr}\left(\mathbf{G}_{k} \mathbf{R}_{\mathbf{n}_{k}} \mathbf{G}_{k}^{T}\right) .
\end{aligned}
$$

In this work, we strive to minimize the maximum MSE over all users under the optical power constraint (4); that is,

$$
\begin{aligned}
\min _{\left\{\mathbf{W}_{j}\right\}, \mathbf{G}_{k}} & \max _{k=1, \ldots, K} \mathrm{MSE}_{k} \\
\text { s.t. } & \sum_{k=1}^{K}\left\|\mathbf{W}_{k}^{T} \mathbf{e}_{n}\right\|_{1} \leqslant p_{t}, \quad n=1, \ldots, N .
\end{aligned}
$$

However, the objective function is nonconvex because of the multiple optimum variables $\left\{\mathbf{W}_{j}\right\}$ and $\mathbf{G}_{k}$. As a result, the alternating minimization approach is introduced and described briefly in the following steps.

(a) By fixing the precoders $\left\{\mathbf{W}_{j}\right\}$, the optimal equalizer $\mathbf{G}_{k}^{*}$ can be obtained by taking the differential of the objective function (11a) with respect to $\mathbf{G}_{k}$; that is,

$$
\mathbf{G}_{k}^{*}=\frac{\mathbf{R}_{\mathbf{s}_{k}}\left(\mathbf{H}_{k} \mathbf{W}_{k}\right)^{T}}{\sum_{j=1}^{K} \mathbf{H}_{k} \mathbf{W}_{j} \mathbf{R}_{\mathbf{s}_{j}}\left(\mathbf{H}_{k} \mathbf{W}_{j}\right)^{T}+\mathbf{R}_{\mathbf{n}_{k}}} .
$$

(b) From the relationship between the Kronecker product and the vector operator $\operatorname{vec}(\mathbf{B A C})=\left(\mathbf{C}^{T} \otimes \mathbf{B}\right) \operatorname{vec}(\mathbf{A})$, the first term of the MSE function (10) can be reformulated as a quadratic form; that is,

$$
\begin{aligned}
& \operatorname{tr}\left(\mathbf{G}_{k} \mathbf{H}_{k} \mathbf{W}_{j} \mathbf{R}_{\mathbf{s}_{j}}\left(\mathbf{G}_{k} \mathbf{H}_{k} \mathbf{W}_{j}\right)^{T}\right) \\
& =\operatorname{tr}\left(\mathbf{W}_{j} \mathbf{R}_{\mathbf{s}_{j}} \mathbf{W}_{j}^{T} \mathbf{H}_{k}^{T} \mathbf{G}_{k}^{T} \mathbf{G}_{k} \mathbf{H}_{k}\right) \\
& \quad=\operatorname{vec}\left(\mathbf{W}_{j}^{T}\right)^{T} \operatorname{vec}\left(\mathbf{R}_{\mathbf{s}_{j}} \mathbf{W}_{j}^{T} \mathbf{H}_{k}^{T} \mathbf{G}_{k}^{T} \mathbf{G}_{k} \mathbf{H}_{k}\right) \\
& =\operatorname{vec}\left(\mathbf{W}_{j}^{T}\right)^{T}\left(\mathbf{H}_{k}^{T} \mathbf{G}_{k}^{T} \mathbf{G}_{k} \mathbf{H}_{k} \otimes \mathbf{R}_{\mathbf{s}_{j}}\right) \operatorname{vec}\left(\mathbf{W}_{j}^{T}\right) .
\end{aligned}
$$

(c) Assuming that the maximum MSE value of all $\mathrm{K}$ users is $\varepsilon$ and combining (10) and (13), the optimization problem (11a) and (11b) can be rewritten as follows:

$$
\begin{array}{ll}
\min _{\left\{\mathbf{W}_{j}\right\}, \mathcal{\varepsilon}} & \mathcal{E} \\
\text { s.t. } & \sum_{j=1}^{K} \operatorname{vec}\left(\mathbf{W}_{j}^{T}\right)^{T}\left(\mathbf{H}_{k}^{T} \mathbf{G}_{k}^{T} \mathbf{G}_{k} \mathbf{H}_{k} \otimes \mathbf{R}_{\mathbf{s}_{j}}\right) \operatorname{vec}\left(\mathbf{W}_{j}^{T}\right)-\operatorname{tr}\left(\mathbf{G}_{k} \mathbf{H}_{k} \mathbf{W}_{k} \mathbf{R}_{\mathbf{s}_{k}}\right)-\operatorname{tr}\left(\mathbf{R}_{\mathbf{s}_{k}}\left(\mathbf{G}_{k} \mathbf{H}_{k} \mathbf{W}_{k}\right)^{T}\right)+\operatorname{tr}\left(\mathbf{R}_{\mathbf{s}_{k}}\right) \\
& +\operatorname{tr}\left(\mathbf{G}_{k} \mathbf{R}_{\mathbf{n}_{k}} \mathbf{G}_{k}^{T}\right) \leqslant \varepsilon, \quad k=1, \ldots, K \\
& \sum_{k=1}^{K}\left\|\mathbf{W}_{k}^{T} \mathbf{e}_{n}\right\|_{1} \leqslant p_{t}, \quad n=1, \ldots, N
\end{array}
$$

which is convex with respect to $\left\{\mathbf{W}_{j}\right\}$ and can be solved directly by CVX. Details of the reformulation process can be found in Appendix A.

The iterative algorithm is stopped when the difference between the optimal solutions of two successive iterations is less than a predefined threshold or the iteration number reaches the predefined default maximum number.

Each step of the proposed approach is summarized in Algorithm 1 explicitly.

\section{Robust Transceiver Design with Imperfect Channel State Information}

In practice, the CSI may not be perfectly known and the estimation errors may exist which could lead to the severe degradation of system performance. As a result, it is necessary to develop a robust design against imperfect CSI over the indoor MU-MIMO VLC systems.

Considering the channel imperfection, the channel can be expressed as

$$
\mathbf{H}=\widehat{\mathbf{H}}+\Delta \mathbf{H},
$$

where $\widehat{\mathbf{H}} \in \mathbb{R}^{M \times N}$ and $\Delta \mathbf{H} \in \mathbb{R}^{M \times N}$ represent the estimated channel information and the channel uncertainty, respectively. A Gaussian-Kronecker model is used to present the channel uncertainty $\Delta \mathbf{H}$; that is,

$$
\Delta \mathbf{H} \sim \mathcal{N}\left(0, \sigma_{e}^{2} \boldsymbol{\Sigma} \otimes \Psi\right)
$$

with the variance $\sigma_{e}^{2}$ of channel imperfection, the row covariance matrix $\Sigma \in \mathbb{R}^{M \times M}$, and the column covariance matrix $\Psi \in \mathbb{R}^{N \times N}$. Hence, the channel matrix $\mathbf{H}$ satisfies the same model given by $\mathbf{H} \sim \mathcal{N}\left(\widehat{\mathbf{H}}, \sigma_{e}^{2} \boldsymbol{\Sigma} \otimes \Psi\right)$, which has a property of matrix variate normal distribution [20]; that is,

$$
\mathbb{E}_{\mathbf{H}}\left(\mathbf{H} \mathbf{A} \mathbf{H}^{T}\right)=\sigma_{e}^{2} \operatorname{tr}\left(\mathbf{A}^{T} \boldsymbol{\Psi}\right) \boldsymbol{\Sigma}+\widehat{\mathbf{H}} \mathbf{A} \widehat{\mathbf{H}}^{T},
$$

with any constant matrix $\mathbf{A} \in \mathbb{R}^{N \times N}$. 
(1) Initialize precoders $\left\{\mathbf{W}_{j}^{0}\right\}$ within constraint (4) and set the DC bias $\mathbf{p}$. Let $i=0$.

(2) Repeat:

(3) Update $\mathbf{G}_{k}^{i}$ with given $\left\{\mathbf{W}_{j}^{i}\right\}$ according to (12);

(4) With $\mathbf{G}_{k}^{i}$, solve problem (14a), (14b), and (14c) by using CVX to obtain the optimal precoders $\left\{\mathbf{W}_{j}^{i+1}\right\}$;

(5) $i=i+1$.

(6) Until $\left\|\mathbf{W}_{k}^{i}-\mathbf{W}_{k}^{i-1}\right\|_{\mathrm{F}}^{2} \leqslant \epsilon$ or $i=i_{\max }$, where $\epsilon$ is a predefined threshold and $i_{\max }$ is the predefined default max iteration number.

Algorithm 1: Iterative algorithm for joint design of precoders and equalizers.

Under the assumption of channel imperfection, the MSE can be written as follows:

$$
\begin{aligned}
\widetilde{\operatorname{MSE}}_{k}\left(\mathbf{r}_{k}, \mathbf{s}_{k},\left\{\mathbf{W}_{j}\right\}_{j=1, \ldots, k, \ldots, K}, \mathbf{G}_{k}\right) \\
=\mathbb{E}_{\mathbf{s}_{k}, \mathbf{n}_{k}, \mathbf{H}_{k}}\left\{\left\|\mathbf{r}_{k}-\mathbf{G}_{k} \widehat{\mathbf{H}}_{k} \mathbf{p}-\mathbf{s}_{k}\right\|_{\mathrm{F}}^{2}\right\} \\
=\mathbb{E}_{\mathbf{H}_{k}}\left\{\sum_{j=1}^{K} \operatorname{tr}\left(\mathbf{G}_{k} \mathbf{H}_{k} \mathbf{W}_{j} \mathbf{R}_{\mathbf{s}_{j}} \mathbf{W}_{j}^{T} \mathbf{H}_{k}^{T} \mathbf{G}_{k}^{T}\right)+\operatorname{tr}\left(\mathbf{R}_{\mathbf{s}_{k}}\right)\right. \\
-\operatorname{tr}\left(\mathbf{G}_{k} \mathbf{H}_{k} \mathbf{W}_{k} \mathbf{R}_{\mathbf{s}_{k}}\right)-\operatorname{tr}\left(\mathbf{R}_{\mathbf{s}_{k}}\left(\mathbf{G}_{k} \mathbf{H}_{k} \mathbf{W}_{k}\right)^{T}\right) \\
\left.+\operatorname{tr}\left(\mathbf{G}_{k} \Delta \mathbf{H}_{k} \mathbf{p} \mathbf{p}^{T} \Delta \mathbf{H}_{k}^{T} \mathbf{G}_{k}^{T}\right)+\operatorname{tr}\left(\mathbf{G}_{k} \mathbf{R}_{\mathbf{n}_{k}} \mathbf{G}_{k}^{T}\right)\right\} \\
=\sum_{j=1}^{K} \sigma_{e}^{2} \operatorname{tr}\left(\mathbf{G}_{k} \operatorname{tr}\left(\mathbf{W}_{j} \mathbf{R}_{\mathbf{s}_{j}} \mathbf{W}_{j}^{T} \mathbf{\Psi}\right) \mathbf{\Sigma} \mathbf{G}_{k}^{T}\right) \\
+\sum_{j=1}^{K} \operatorname{tr}\left(\mathbf{G}_{k} \widehat{\mathbf{H}}_{k} \mathbf{W}_{j} \mathbf{R}_{\mathbf{s}_{j}} \mathbf{W}_{j}^{T} \widehat{\mathbf{H}}_{k}^{T} \mathbf{G}_{k}^{T}\right)+\operatorname{tr}\left(\mathbf{R}_{\mathbf{s}_{k}}\right) \\
+\operatorname{tr}\left(\mathbf{G}_{k} \widehat{\mathbf{H}}_{k} \mathbf{W}_{k} \mathbf{R}_{\mathbf{s}_{k}}\right)-\operatorname{tr}\left(\mathbf{R}_{\mathbf{s}_{k}}\left(\mathbf{G}_{k} \widehat{\mathbf{H}}_{k} \mathbf{W}_{k}\right)^{T}\right) \\
+\sigma_{e}^{2} \operatorname{tr}\left(\mathbf{G}_{k} \operatorname{tr}\left(\mathbf{p} \mathbf{p}^{T} \boldsymbol{\Psi}\right) \mathbf{\Sigma} \mathbf{G}_{k}^{T}\right)+\operatorname{tr}\left(\mathbf{G}_{k} \mathbf{R}_{\mathbf{n}_{k}} \mathbf{G}_{k}^{T}\right)
\end{aligned}
$$

The IUI can be suppressed by minimizing the maximum MSE under the power constraint; that is,

$$
\begin{aligned}
\min _{\left\{\mathbf{W}_{j}\right\}, \mathbf{G}_{k}} & \max _{k=1, \ldots, K} \widetilde{\mathrm{MSE}}_{k} \\
\text { s.t. } & \sum_{k=1}^{K}\left\|\mathbf{W}_{k}^{T} \mathbf{e}_{n}\right\|_{1} \leqslant p_{t}, \quad n=1, \ldots, N,
\end{aligned}
$$

which is nonconvex due to the simultaneous optimization of $\left\{\mathbf{W}_{j}\right\}$ and $\mathbf{G}_{k}$. Following the iterative algorithm in the perfect CSI scenario, the optimal solutions can be achieved by the following steps.

(a) With the fixed $\left\{\mathbf{W}_{j}\right\}$, the equalizer $\mathbf{G}_{k}^{*}$ for $k$ th user can similarly rewritten as

$$
\mathbf{G}_{k}^{*}=\mathbf{R}_{\mathbf{s}_{k}}\left(\widehat{\mathbf{H}}_{k} \mathbf{W}_{k}\right)^{T}\left(\mathbf{Q}+\sigma_{e}^{2} \operatorname{tr}\left(\mathbf{p} \mathbf{p}^{T} \boldsymbol{\Psi}\right) \boldsymbol{\Sigma}+\mathbf{R}_{\mathbf{n}_{k}}\right)^{-1}
$$

where

$$
\mathbf{Q}=\sum_{j=1}^{K}\left(\sigma_{e}^{2} \operatorname{tr}\left(\mathbf{W}_{j} \mathbf{R}_{\mathbf{s}_{j}} \mathbf{W}_{j}^{T} \Psi\right) \boldsymbol{\Sigma}+\widehat{\mathbf{H}}_{k} \mathbf{W}_{j} \mathbf{R}_{\mathbf{s}_{j}} \mathbf{W}_{j}^{T} \widehat{\mathbf{H}}_{k}^{T}\right) .
$$

(b) Under a similar transformation, the first and second terms of (18) can be rewritten as

$$
\begin{aligned}
& \operatorname{tr}\left(\mathbf{G}_{k} \operatorname{tr}\left(\mathbf{W}_{j} \mathbf{R}_{\mathbf{s}_{j}} \mathbf{W}_{j}^{T} \boldsymbol{\Psi}\right) \boldsymbol{\Sigma} \mathbf{G}_{k}^{T}\right) \\
& =\operatorname{tr}\left(\mathbf{W}_{j} \mathbf{R}_{\mathbf{s}_{j}} \mathbf{W}_{j}^{T} \boldsymbol{\Psi}\right) \operatorname{tr}\left(\mathbf{G}_{k} \boldsymbol{\Sigma} \mathbf{G}_{k}^{T}\right) \\
& \quad=\operatorname{vec}\left(\mathbf{W}_{j}^{T}\right)^{T}\left(\boldsymbol{\Psi}^{T} \otimes \mathbf{R}_{\mathbf{s}_{j}}\right) \operatorname{vec}\left(\mathbf{W}_{j}^{T}\right) \operatorname{tr}\left(\mathbf{G}_{k} \boldsymbol{\Sigma} \mathbf{G}_{k}^{T}\right), \\
& \operatorname{tr}\left(\mathbf{G}_{k} \widehat{\mathbf{H}}_{k} \mathbf{W}_{j} \mathbf{R}_{\mathbf{s}_{j}} \mathbf{W}_{j}^{T} \widehat{\mathbf{H}}_{k}^{T} \mathbf{G}_{k}^{T}\right) \\
& \quad=\operatorname{vec}\left(\mathbf{W}_{j}^{T}\right)^{T}\left(\widehat{\mathbf{H}}_{k}^{T} \mathbf{G}_{k}^{T} \mathbf{G}_{k} \widehat{\mathbf{H}}_{k} \otimes \mathbf{R}_{\mathbf{s}_{j}}\right) \operatorname{vec}\left(\mathbf{W}_{j}^{T}\right),
\end{aligned}
$$

respectively.

(c) Introducing the maximum MSE value $\varepsilon$ of $\mathrm{K}$ users and substituting (22) and (23) into (18), the robust max-MSE minimization problem can be presented in (24a), (24b), and (24c) and solved via the CVX tool. A detailed reformulation process can be found in Appendix B.

$$
\begin{array}{ll}
\min _{\left\{\mathbf{W}_{j}\right\}} & \mathcal{E} \\
\text { s.t. } & \sum_{j=1}^{K} \sigma_{e}^{2} \operatorname{vec}\left(\mathbf{W}_{j}^{T}\right)^{T}\left(\Psi^{T} \otimes \mathbf{R}_{\mathbf{s}_{j}}\right) \operatorname{vec}\left(\mathbf{W}_{j}^{T}\right) \operatorname{tr}\left(\mathbf{G}_{k} \mathbf{\Sigma} \mathbf{G}_{k}^{T}\right)+\sum_{j=1}^{K} \operatorname{vec}\left(\mathbf{W}_{j}^{T}\right)^{T}\left(\widehat{\mathbf{H}}_{k}^{T} \mathbf{G}_{k}^{T} \mathbf{G}_{k} \widehat{\mathbf{H}}_{k} \otimes \mathbf{R}_{\mathbf{s}_{j}}\right) \operatorname{vec}\left(\mathbf{W}_{j}^{T}\right) \\
& +\operatorname{tr}\left(\mathbf{R}_{\mathbf{s}_{k}}\right)-\operatorname{tr}\left(\mathbf{G}_{k} \widehat{\mathbf{H}}_{k} \mathbf{W}_{k} \mathbf{R}_{\mathbf{s}_{k}}\right)-\operatorname{tr}\left(\mathbf{R}_{\mathbf{s}_{k}}\left(\mathbf{G}_{k} \widehat{\mathbf{H}}_{k} \mathbf{W}_{k}\right)^{T}\right)+\sigma_{e}^{2} \operatorname{tr}\left(\mathbf{G}_{k} \operatorname{tr}\left(\mathbf{p} \mathbf{p}^{T} \boldsymbol{\Psi}\right) \mathbf{\Sigma} \mathbf{G}_{k}^{T}\right)+\operatorname{tr}\left(\mathbf{G}_{k} \mathbf{R}_{\mathbf{n}_{k}} \mathbf{G}_{k}^{T}\right) \leqslant \varepsilon \\
& \sum_{k=1}^{K}\left\|\mathbf{W}_{k}^{T} \mathbf{e}_{n}\right\|_{1} \leqslant p_{t}, \quad n=1, \ldots, N .
\end{array}
$$


TABLE 1: Simulation parameters.

\begin{tabular}{lc}
\hline Description & Value \\
\hline Number of LEDs per array & 3600 \\
LED pitch & $0.01 \mathrm{~m}$ \\
Data rate & $100 \mathrm{Mbit} / \mathrm{s}$ \\
Modulation & OOK \\
LED array pitch & $2 \mathrm{~m}$ \\
LED optical linear region & {$[0,100 \mathrm{~W}]$} \\
Transmitter semiangle & $70 \mathrm{deg}$ \\
Receiver field of view (half angle) & $70 \mathrm{deg}$ \\
Number of PDs per user terminal & 2 \\
Refractive index of concentrator, $\kappa$ & 1.5 \\
Physical area of PD, $A$ & $1.0 \mathrm{~cm}^{2}$ \\
PD pitch & $0.01 \mathrm{~m}$ \\
\hline
\end{tabular}

TABLE 2: The location examples of two users (m).

\begin{tabular}{ll}
\hline Case 1 & {$[2.5,2.5,0.8],[4.9,4.9,0.8]$} \\
Case 2 & {$[1.5,1.5,0.8],[3.5,3.5,0.8]$} \\
Case 3 & {$[2.5,2.5,0.8],[3.5,3.5,0.8]$} \\
\hline
\end{tabular}

The iterative process continues until it converges or the maximum predefined number is achieved.

\section{Simulation Results}

This section presents the simulation results of the proposed design under a $5 \times 5 \times 3 \mathrm{~m}^{3}$ inner part of a large indoor space environment. There are four LED arrays on the ceiling with the positions [1.5, 1.5. 3], [3.5, 1.5, 3], [1.5, 3.5, 3], [3.5, 2.5, 3] and two stationary users in the room. The entries of the covariance matrices in (16) can be chosen as [16]

$$
\begin{aligned}
& {[\Psi]_{i, j}=\alpha^{|i-j|}, \quad i, j=1, \ldots, N,} \\
& {[\Sigma]_{i, j}=\beta^{|i-j|}, \quad i, j=1, \ldots, M,}
\end{aligned}
$$

where $0<\alpha, \beta<1$ are correlation coefficients. Unless otherwise specified, the rest of the parameters used and the user location examples are specified in Tables 1 and 2, respectively.

5.1. Convergence Performance. The convergence of the proposed iterative algorithm is presented in Figure 2 with the location of case 2 . The optical power constraint is set as $p_{n}=10 \mathrm{~W}$, and other parameters are set as $\alpha=0.5, \beta=$ $0.6, \sigma_{s}^{2}=1$, and $\sigma_{n}^{2}=1$. The proposed iterative algorithm converges to the optimum point with limited iterations. It is obvious that the sum-MSE performance with perfect CSI is better than the one with imperfect CSI under the same power constraint.

5.2. Robustness Performance. Figure 3 investigates the robustness of the proposed algorithm with the same parameters. Due to the fact that the channel coefficients between arrayed LED transmitters and receivers are in the order of $10^{-1}$, the estimation error variances can be chosen from $10^{-4}$ to

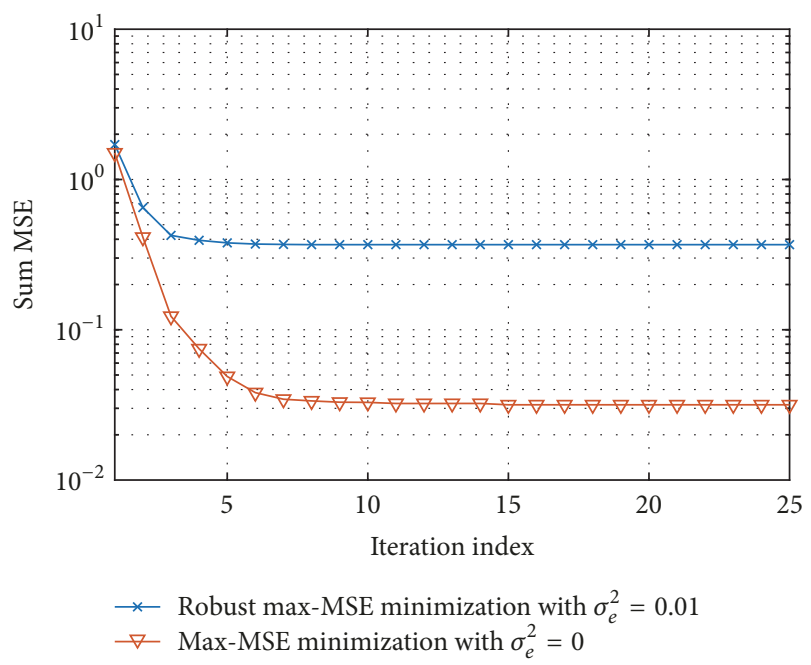

FIGURE 2: Convergence performance of the proposed iterative algorithm.

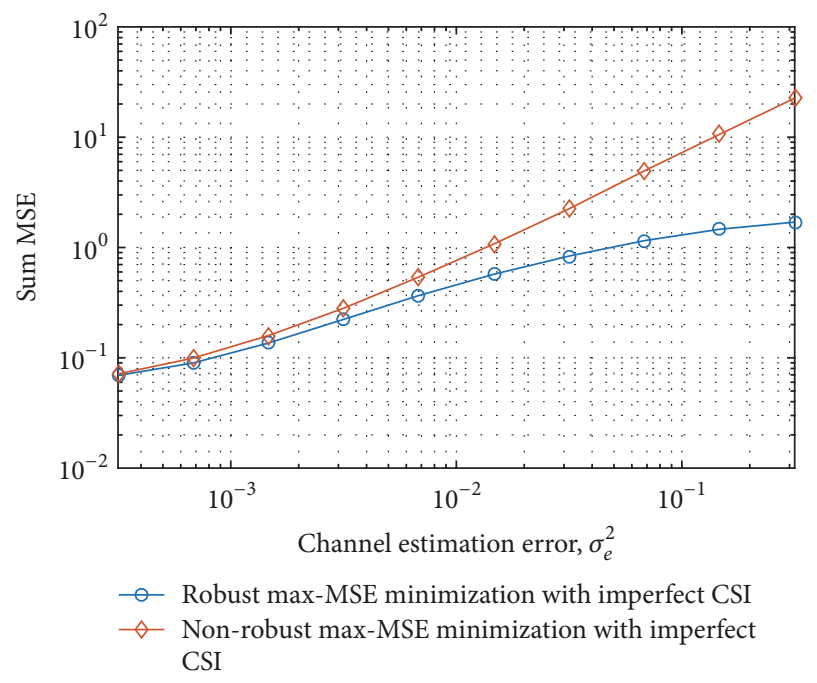

FIGURE 3: The robustness of the proposed algorithm with $\alpha=0.5$, $\beta=0.6$, and $p_{n}=10 \mathrm{~W}$.

1 for this simulation. When the channel estimation error $\sigma_{e}^{2}$ is small, the robust scheme and the nonrobust scheme have almost the same performance. With the increase of the channel estimation error, the sum MSE of the robust scheme and the nonrobust scheme increase. However, the robust scheme outperforms the one without considering the channel imperfections. It is concluded that the MSE performance is sensitive to the large CSI error and the robust scheme can provide robustness against the channel uncertainties.

5.3. MSE Fairness Performance. The sum-MSE performances over case 2 are illustrated in Figure 4, where the short forms "min-max-MSE," "min-sum-MSE," and "BD scheme" represent the proposed max-MSE minimization approach, the sum-MSE minimization algorithm [12], and the maxMSE minimization scheme with the assistance of block 


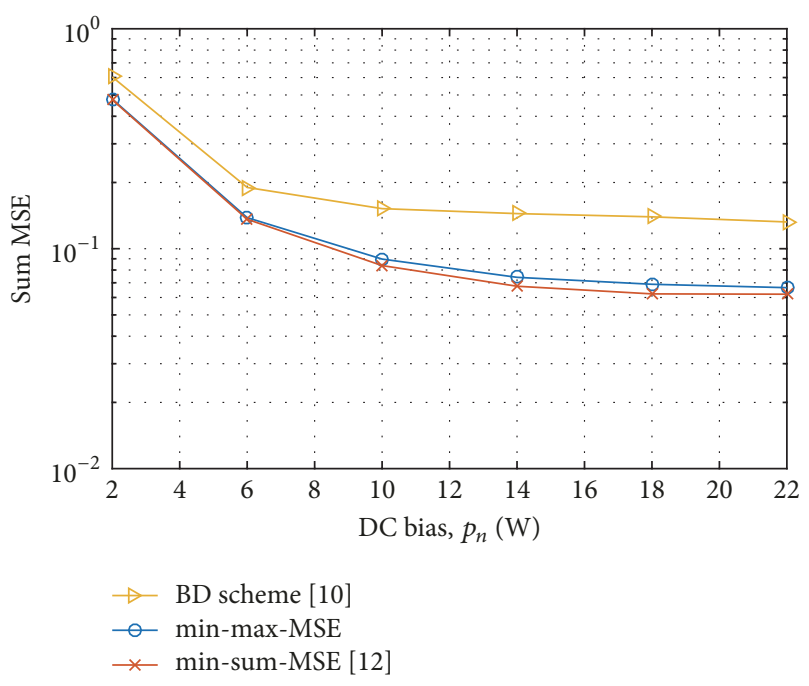

FIGURE 4: Sum MSE versus optical power.

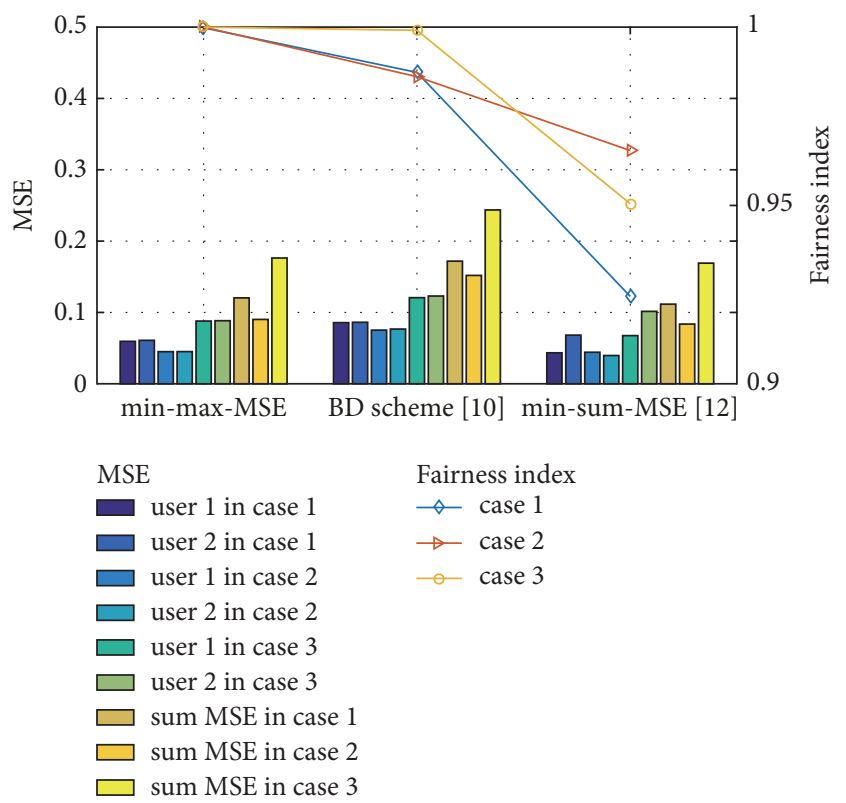

FIGURE 5: MSE distribution and fairness performance of different algorithms.

diagonalization (BD) algorithm [10], respectively. The simulation parameters are set as $\alpha=0.5, \beta=0.6$, and $\sigma_{e}^{2}=0.001$. It is shown that the sum-MSE minimization algorithm can achieve the lowest sum MSE since it is designed to minimize the total MSE without any fairness constraints, while the proposed max-MSE minimization approach sacrifices a little MSE to balance the fairness. On the other hand, the BD scheme has the highest sum MSE since it suppresses the interference by imposing the IUI on the channel's null space under the limited degrees of freedom.

Furthermore, Figure 5 demonstrates the fairness superiority via the MSE distribution performances with the three mentioned algorithms. Three location configurations

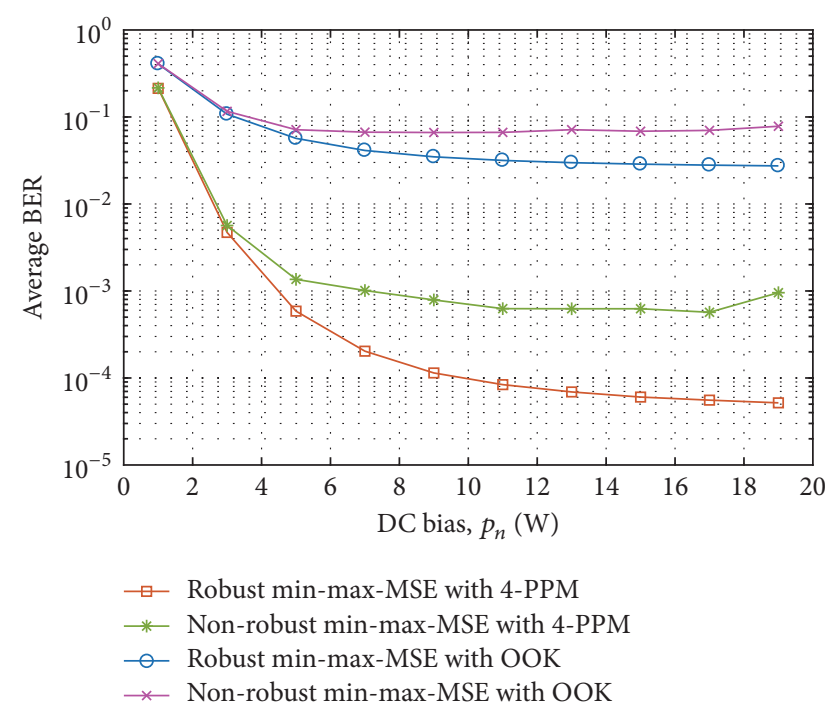

FIGURE 6: BER performance of the different modulation schemes with $\sigma_{e}^{2}=0.005$.

specified in Table 2 are considered with $p_{n}=10 \mathrm{~W}$. Jain's fairness index is adopted as the criterion to compare the fairness, which is defined as $f(\mathbf{x})=\left(\sum_{i=1}^{K} x_{i}\right)^{2} /\left(K \sum_{i=1}^{K} x_{i}^{2}\right)$ with $x_{i} \geqslant 0$ [21]. The value of $f(\mathbf{x})$ indicates the fairness of the system. More specifically, the maximum fairness index is 1 when the resources are allocated equally among all users. If only one user dominates the resource while others receive none, the fairness index will reduce to $1 / K$. Therefore, the fairness index is bounded between the intervals of $[1 / K, 1]$. In Figure 5, the proposed min-max-MSE algorithm performs slightly worse than the min-sum-MSE algorithm in terms of sum MSE, but it offers fairer transmission for different location settings. The fairness index of the proposed minmax-MSE algorithm is highest, which indicates that the proposed algorithm provides the best fairness performance among the three approaches.

5.4. BER Performance. In our simulations, OOK modulation is applied and the theoretical BER can be calculated by [22]

$$
\mathrm{BER}=\mathrm{Q}(\sqrt{\mathrm{SINR}}),
$$

where SINR $=\left(\sum \mathrm{MSE}_{k}\right)^{-1}-1[23]$ and $Q(\cdot)$ represents the $Q$-function. Figures 6 and 7 investigate the BER performance with the same parameters in Section 5.3. The BER performances of OOK modulation and 4-level pulseposition modulation (4-PPM) are compared in Figure 6. The BER decreases with the increase of the optical power, and the robust scheme performs better than the nonrobust scheme. The curves of nonrobust scheme become flat and the gaps between robust scheme and nonrobust scheme become larger. This is because, without considering the channel imperfection, the DC bias estimation error term $\sigma_{e}^{2} \operatorname{tr}\left(\mathbf{G}_{k} \operatorname{tr}\left(\mathbf{p p}^{T} \boldsymbol{\Psi}\right) \boldsymbol{\Sigma} \mathbf{G}_{k}^{T}\right)$ increases when optical power $\mathbf{p}$ becomes larger. The PPM modulation technique improves the power efficiency of the OOK modulation to achieve a given 


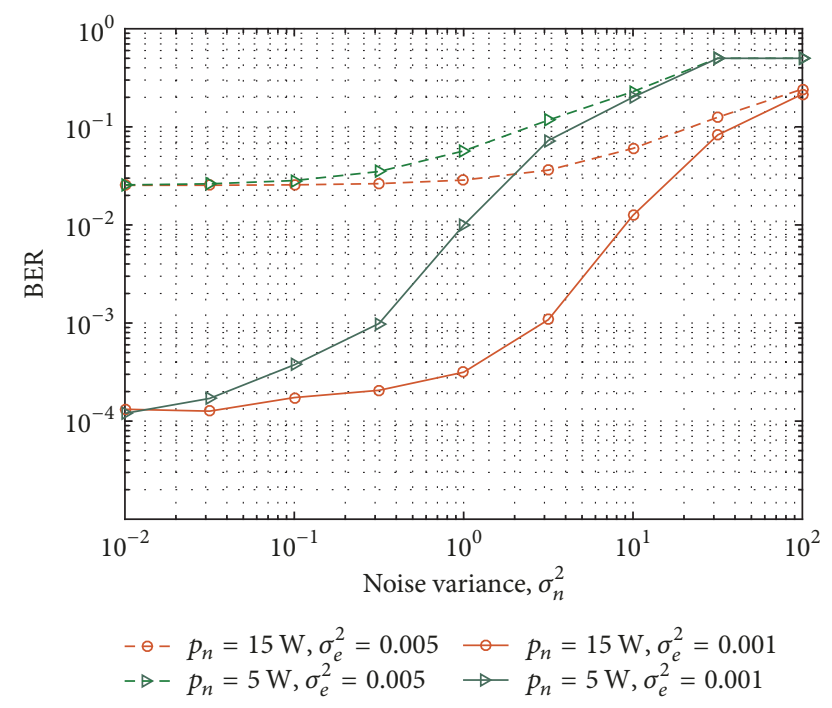

FIGURE 7: BER performance of the robust design with different noise variances.

BER. On the other hand, the PPM modulation requires both the slot and the symbol-level synchronization which may increase the receiver complexity, while the OOK modulation is simple to be implemented, leading to its wide use in optical wireless systems.

Figure 7 presents the BER performance with various noise variance values to further investigate the performance of the proposed robust design with the OOK modulation. As we expected, the BER decreases with the reduction of noise variance. With fixed noise variance, the BER with higher optical power is better than that with the lower power. It is clear that, under the low noise variance, the BER is only dominated by the channel uncertainties, and its performance is degraded with the large channel imperfection.

5.5. The Impact of Different Geometric Metrics on System Performance. Finally, we investigate the impact of different geometric metrics, including users' relative positions, heights, and angles in Figures 8 and 9. It is assumed that one user is fixed at the position of $[2.5,2.5,0.8]$ and another user moves around on the same receive plane. As expected, the MSE performance becomes worse when two users are getting closer, shown in Figure 8, due to the negative channel correlation.

Besides, Figure 9 illustrates the MSE performance with varying height of the receiver plane and receiver's FOV. The users' horizontal coordinates are set as $[2.5,2.5]$ and $[4.5,4.5]$. In this situation, it is nearly impossible to receive optical signals with small angles or on the plane of high level where the communication link is interrupted. When the FOV is large enough to receive all the transmitted optical links, it is hard to distinguish the signals for different users in the receivers, which reduces the ability for signal recovery and degrades the MSE performance.

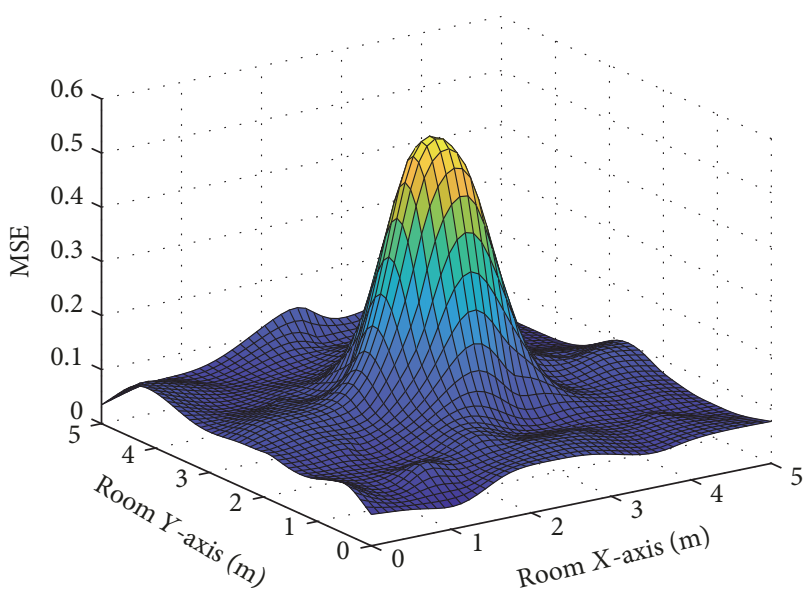

FIGURE 8: MSE distribution of different positions.

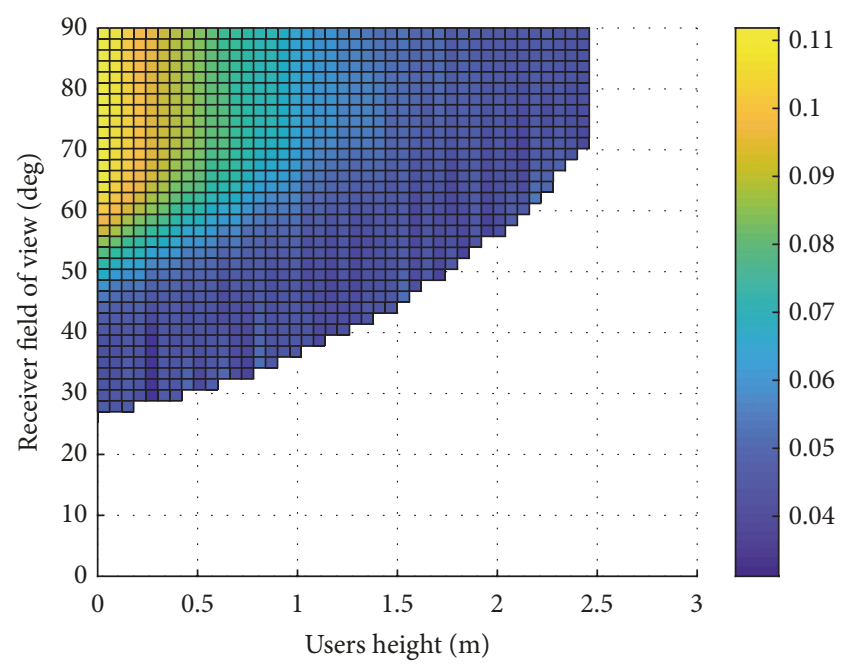

FIGURE 9: Impact of user's height and angles on MSE performance.

\section{Conclusion}

In this work, the optimal transceivers are designed for the indoor MU-MIMO VLC system where the max-MSE minimization approach is developed to suppress the IUI. Due to the channel uncertainties in practice, the mismatch between the estimated channel information and the perfect channel information may severely degrade the system performance. Therefore, the robust design is investigated against the channel uncertainties. The simulation results demonstrate that the robust design can provide robustness against the channel imperfections and gains a considerable BER performance. Moreover, the proposed algorithm is verified to achieve almost the same performance of the sum-MSE minimization algorithm, but guaranteeing the fairness among the multiple users. The influences of different geometric metrics are also discussed in simulation results, and it is demonstrated that the channel correlation, the distance between transmitters and receivers, and the receiver FOV are the decisive factors for performance improvement. 


\section{Appendix}

\section{A. Formulation of Problem (14a), $(14 b)$, and $(14 c)$}

By introducing an auxiliary variable $\varepsilon$ that serves an upper bound on the MSE value of $k$ th user, the optimization problem (11a) and (11b) can be transformed as

$$
\begin{array}{ll}
\min _{\left\{\mathbf{W}_{j}\right\}, \varepsilon} & \varepsilon \\
\text { s.t. } & \operatorname{MSE}_{k} \leqslant \varepsilon, \quad k=1, \ldots, K, \\
& \sum_{k=1}^{K}\left\|\mathbf{W}_{k}^{T} \mathbf{e}_{n}\right\|_{1} \leqslant p_{t} \quad, n=1, \ldots, N .
\end{array}
$$
ten as

Using the transformation of (13), the MSE can be rewrit-

$$
\begin{aligned}
& \mathrm{MSE}_{k} \\
& =\sum_{j=1}^{K} \operatorname{tr}\left(\mathbf{G}_{k} \mathbf{H}_{k} \mathbf{W}_{j} \mathbf{R}_{\mathbf{s}_{j}}\left(\mathbf{G}_{k} \mathbf{H}_{k} \mathbf{W}_{j}\right)^{T}\right)+\operatorname{tr}\left(\mathbf{R}_{\mathbf{s}_{k}}\right) \\
& \quad-\operatorname{tr}\left(\mathbf{G}_{k} \mathbf{H}_{k} \mathbf{W}_{k} \mathbf{R}_{\mathbf{s}_{k}}\right)-\operatorname{tr}\left(\mathbf{R}_{\mathbf{s}_{k}}\left(\mathbf{G}_{k} \mathbf{H}_{k} \mathbf{W}_{k}\right)^{T}\right) \\
& \quad+\operatorname{tr}\left(\mathbf{G}_{k} \mathbf{R}_{\mathbf{n}_{k}} \mathbf{G}_{k}^{T}\right) \\
& =\sum_{j=1}^{K} \operatorname{vec}\left(\mathbf{W}_{j}^{T}\right)^{T}\left(\mathbf{H}_{k}^{T} \mathbf{G}_{k}^{T} \mathbf{G}_{k} \mathbf{H}_{k} \otimes \mathbf{R}_{\mathbf{s}_{j}}\right) \operatorname{vec}\left(\mathbf{W}_{j}^{T}\right) \\
& \quad-\operatorname{tr}\left(\mathbf{G}_{k} \mathbf{H}_{k} \mathbf{W}_{k} \mathbf{R}_{\mathbf{s}_{k}}\right)-\operatorname{tr}\left(\mathbf{R}_{\mathbf{s}_{k}}\left(\mathbf{G}_{k} \mathbf{H}_{k} \mathbf{W}_{k}\right)^{T}\right) \\
& \quad+\operatorname{tr}\left(\mathbf{G}_{k} \mathbf{R}_{\mathbf{n}_{k}} \mathbf{G}_{k}^{T}\right)+\operatorname{tr}\left(\mathbf{R}_{\mathbf{s}_{k}}\right) .
\end{aligned}
$$

Applying (A.2) on (A.1a), (A.1b), and (A.1c), we can obtain optimization problem (14a), (14b), and (14c).

\section{B. Formulation of Problem (24a), (24b), and (24c)}

Similar to the formulation of problem (14a), (14b), and (14c), the robust max-MSE minimization problem (19a) and (19b) can be rewritten as

$$
\begin{array}{ll}
\min _{\left\{\mathbf{W}_{j}\right\}, \mathcal{\varepsilon}} & \varepsilon \\
\text { s.t. } & \widetilde{\operatorname{MSE}}_{k} \leqslant \varepsilon, \quad k=1, \ldots, K, \\
& \sum_{k=1}^{K}\left\|\mathbf{W}_{k}^{T} \mathbf{e}_{n}\right\|_{1} \leqslant p_{t}, \quad n=1, \ldots, N,
\end{array}
$$

where the corresponding MSE can be reformed as

$$
\begin{aligned}
& \widetilde{\mathrm{MSE}}_{k} \\
& =\sum_{j=1}^{K} \sigma_{e}^{2} \operatorname{tr}\left(\mathbf{G}_{k} \operatorname{tr}\left(\mathbf{W}_{j} \mathbf{R}_{\mathbf{s}_{j}} \mathbf{W}_{j}^{T} \mathbf{\Psi}\right) \mathbf{\Sigma} \mathbf{G}_{k}^{T}\right) \\
& +\sum_{j=1}^{K} \operatorname{tr}\left(\mathbf{G}_{k} \widehat{\mathbf{H}}_{k} \mathbf{W}_{j} \mathbf{R}_{\mathbf{s}_{j}} \mathbf{W}_{j}^{T} \widehat{\mathbf{H}}_{k}^{T} \mathbf{G}_{k}^{T}\right)+\operatorname{tr}\left(\mathbf{R}_{\mathbf{s}_{k}}\right) \\
& -\operatorname{tr}\left(\mathbf{G}_{k} \widehat{\mathbf{H}}_{k} \mathbf{W}_{k} \mathbf{R}_{\mathbf{s}_{k}}\right)-\operatorname{tr}\left(\mathbf{R}_{\mathbf{s}_{k}}\left(\mathbf{G}_{k} \widehat{\mathbf{H}}_{k} \mathbf{W}_{k}\right)^{T}\right) \\
& +\sigma_{e}^{2} \operatorname{tr}\left(\mathbf{G}_{k} \operatorname{tr}\left(\mathbf{p} \mathbf{p}^{T} \boldsymbol{\Psi}\right) \boldsymbol{\Sigma} \mathbf{G}_{k}^{T}\right)+\operatorname{tr}\left(\mathbf{G}_{k} \mathbf{R}_{\mathbf{n}_{k}} \mathbf{G}_{k}^{T}\right) \\
& =\sum_{j=1}^{K} \operatorname{vec}\left(\mathbf{W}_{j}^{T}\right)^{T}\left(\Psi^{T} \otimes \mathbf{R}_{\mathbf{s}_{j}}\right) \operatorname{vec}\left(\mathbf{W}_{j}^{T}\right) \operatorname{tr}\left(\mathbf{G}_{k} \boldsymbol{\Sigma} \mathbf{G}_{k}^{T}\right) \\
& +\sum_{j=1}^{K} \operatorname{vec}\left(\mathbf{W}_{j}^{T}\right)^{T}\left(\widehat{\mathbf{H}}_{k}^{T} \mathbf{G}_{k}^{T} \mathbf{G}_{k} \widehat{\mathbf{H}}_{k} \otimes \mathbf{R}_{\mathbf{s}_{j}}\right) \operatorname{vec}\left(\mathbf{W}_{j}^{T}\right) \\
& -\operatorname{tr}\left(\mathbf{G}_{k} \widehat{\mathbf{H}}_{k} \mathbf{W}_{k} \mathbf{R}_{\mathbf{s}_{k}}\right)-\operatorname{tr}\left(\mathbf{R}_{\mathbf{s}_{k}}\left(\mathbf{G}_{k} \widehat{\mathbf{H}}_{k} \mathbf{W}_{k}\right)^{T}\right) \\
& +\sigma_{e}^{2} \operatorname{tr}\left(\mathbf{G}_{k} \operatorname{tr}\left(\mathbf{p} \mathbf{p}^{T} \boldsymbol{\Psi}\right) \boldsymbol{\Sigma} \mathbf{G}_{k}^{T}\right)+\operatorname{tr}\left(\mathbf{G}_{k} \mathbf{R}_{\mathbf{n}_{k}} \mathbf{G}_{k}^{T}\right) \\
& +\operatorname{tr}\left(\mathbf{R}_{\mathbf{s}_{k}}\right) \text {, }
\end{aligned}
$$

leading to (24a), (24b), and (24c).

\section{Data Availability}

All relevant data are within the paper.

\section{Conflicts of Interest}

The authors declare that there are no conflicts of interest regarding the publication of this article.

\section{Acknowledgments}

The work of Zhirong Zeng and Huiqin Du is supported by the National Natural Science Foundation of China (Grant no. 61401178) and the Open Research Fund of National Mobile Communications Research Laboratory Southeast University (Grant no. 2017D12). The work of Zujian Wu is supported by the National Natural Science Foundation of China (Grant no. 61602209) and the Fundamental Research Funds for the Central Universities (Grant no. 21615316).

\section{References}

[1] P. H. Pathak, X. Feng, P. Hu, and P. Mohapatra, "Visible light communication, networking, and sensing: a survey, potential and challenges," IEEE Communications Surveys \& Tutorials, vol. 17, no. 4, pp. 2047-2077, 2015.

[2] S. Zhao, "A serial concatenation-based coding scheme for dimmable visible light communication systems," IEEE Communications Letters, vol. 20, no. 10, pp. 1951-1954, 2016. 
[3] R. Shaaban and S. Faruque, "A survey of indoor visible light communication power distribution and color shift keying transmission," in Proceedings of the 2017 IEEE International Conference on Electro Information Technology, EIT 2017, pp. 149-153, Lincoln, Neb, USA, 2017.

[4] M. K. Jha, A. Addanki, Y. V. S. Lakshmi, and N. Kumar, "Channel coding performance of optical MIMO indoor visible light communication," in Proceedings of the International Conference on Advances in Computing, Communications and Informatics, ICACCI 2015, pp. 97-102, Kochi, India, 2015.

[5] K. D. Dambul, D. C. O'Brien, and G. Faulkner, "Indoor optical wireless MIMO system with an imaging receiver," IEEE Photonics Technology Letters, vol. 23, no. 2, pp. 97-99, 2011.

[6] L. Zeng, D. C. O’Brien, H. Le Minh et al., "High data rate Multiple Input Multiple Output (MIMO) optical wireless communications using white LED lighting," IEEE Journal on Selected Areas in Communications, vol. 27, no. 9, pp. 1654-1662, 2009.

[7] H. Marshoud, P. C. Sofotasios, S. Muhaidat, and G. K. Karagiannidis, "Multi-user techniques in visible light communications: A survey," in Proceedings of the International Conference on Advanced Communication Systems and Information Security (ACOSIS), Morocco, 2016.

[8] H. Shen, Y. Deng, W. Xu, and C. Zhao, "Rate-maximized zeroforcing beamforming for VLC multiuser MISO downlinks," IEEE Photonics Journal, vol. 8, no. 1, 2016.

[9] H. Shen, Y. Deng, W. Xu, and C. Zhao, "Rate maximization for downlink multiuser visible light communications," IEEE Access, vol. 4, pp. 6567-6573, 2016.

[10] T. V. Pham, H. Le Minh, Z. Ghassemlooy, T. Hayashi, and A. T. Pham, "Sum-rate maximization of multi-user MIMO visible light communications," in Proceedings of the IEEE International Conference on Communication Workshop, ICCW 2015, pp. 13441349, London, UK, June 2015.

[11] Z. Zeng and H. Du, "Robust precoding scheme for multiuser MIMO visible light communication system," in Proceedings of the 2017 25th European Signal Processing Conference (EUSIPCO), pp. 2546-2550, Kos, Greece, August 2017.

[12] H. Ma, L. Lampe, and S. Hranilovic, "Robust MMSE linear precoding for visible light communication broadcasting systems," in Proceedings of the IEEE Globecom Workshops, GC Wkshps 2013, pp. 1081-1086, Atlanta, Ga, USA, 2013.

[13] B. Li, J. Wang, R. Zhang, H. Shen, C. Zhao, and L. Hanzo, "Multiuser MISO transceiver design for indoor downlink visible light communication under per-LED optical power constraints," IEEE Photonics Journal, vol. 7, no. 4, 2015.

[14] H. Marshoud, D. Dawoud, V. M. Kapinas, G. K. Karagiannidis, S. Muhaidat, and B. Sharif, "MU-MIMO precoding for VLC with imperfect CSI," in Proceedings of the 4th International Workshop on Optical Wireless Communications, IWOW 2015, pp. 93-97, Istanbul, Turkey, 2015.

[15] K.-H. Park, Y.-C. Ko, and M.-S. Alouini, "On the power and offset allocation for rate adaptation of spatial multiplexing in optical wireless MIMO channels," IEEE Transactions on Communications, vol. 61, no. 4, pp. 1535-1543, 2013.

[16] K. Ying, H. Qian, R. J. Baxley, and S. Yao, "Joint Optimization of Precoder and Equalizer in MIMO VLC Systems," IEEE Journal on Selected Areas in Communications, vol. 33, no. 9, pp. 19491958, 2015.
[17] A. Vavoulas, H. G. Sandalidis, T. A. Tsiftsis, and N. Vaiopoulos, "Coverage Aspects of Indoor VLC Networks," Journal of Lightwave Technology, vol. 33, no. 23, Article ID 7299610, pp. 49154921, 2015.

[18] A. Burton, H. Le Minh, Z. Ghassemlooy, E. Bentley, and C. Botella, "Experimental demonstration of $50-\mathrm{Mb} / \mathrm{s}$ visible light communications using $4 \times 4$ MIMO," IEEE Photonics Technology Letters, vol. 26, no. 9, pp. 945-948, 2014.

[19] J. Ding, K. Wang, and Z. Xu, "Impact of different LED-spacing in arrayed LED transmitter on VLC channel modeling," in Proceedings of the 2014 6th International Conference on Wireless Communications and Signal Processing, WCSP 2014, pp. 1-6, Hefei, China, 2014.

[20] A. Gupta and D. Nagar, Matrix Variate Distribution, Chapman \& Hall/CRC, London, UK, 2000.

[21] R. Jain, D. Chiu, and W. Hawe, "A quantitative measure of fairness and discrimination for resource allocation in shared computer systems," Digital Equipment Corporation Research, Technical Report, 1984.

[22] Z. Ghassemlooy, W. Popoola, and S. Rajbhandari, Optical Wireless Communication: System and Channel Modelling with Matlab, CRC publisher, USA, 2012.

[23] D. Pérez Palomar, M. Bengtsson, and B. Ottersten, "Minimum BER linear transceivers for MIMO channels via primal decomposition," IEEE Transactions on Signal Processing, vol. 53, no. 8, pp. 2866-2882, 2005. 


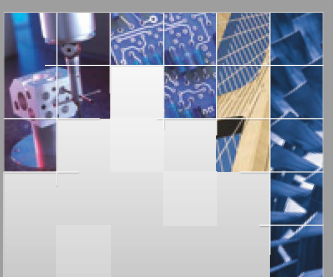

\section{Enfincering}
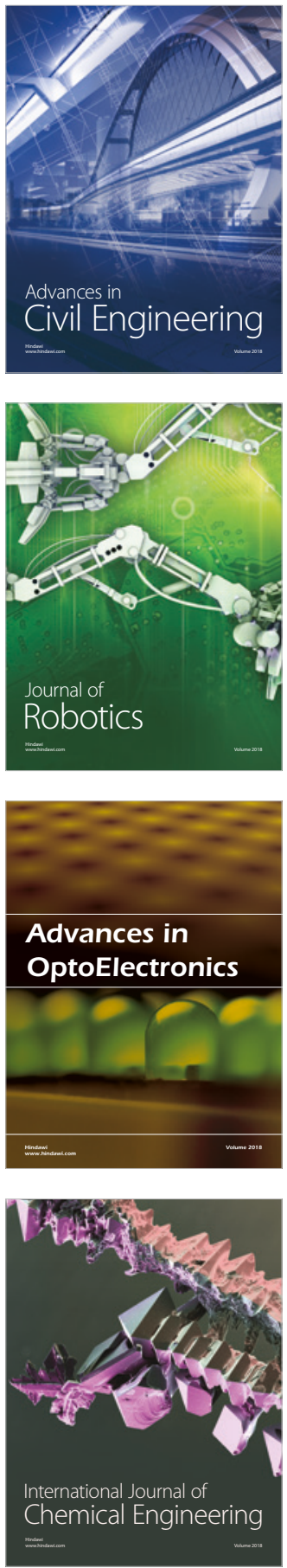

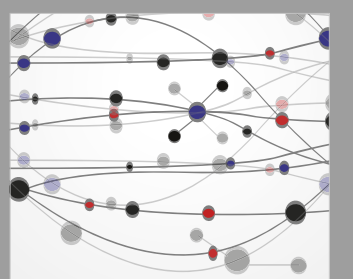

\section{Rotating \\ Machinery}

The Scientific World Journal

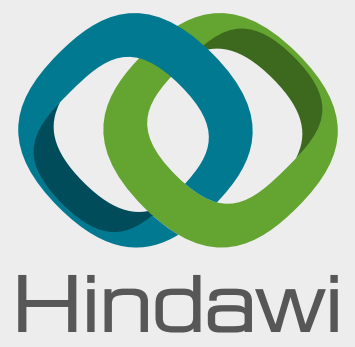

Submit your manuscripts at

www.hindawi.com
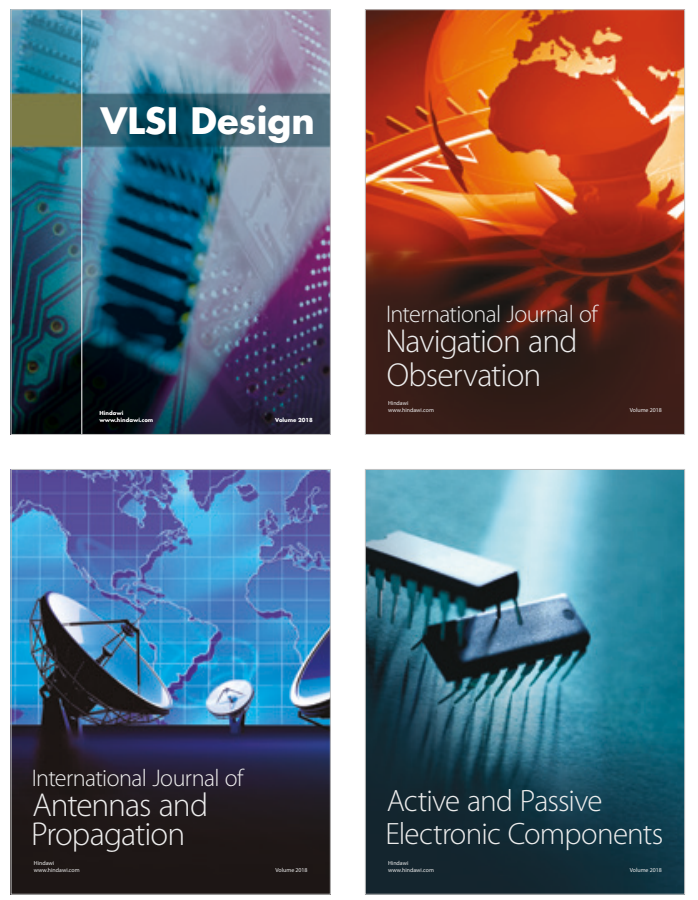
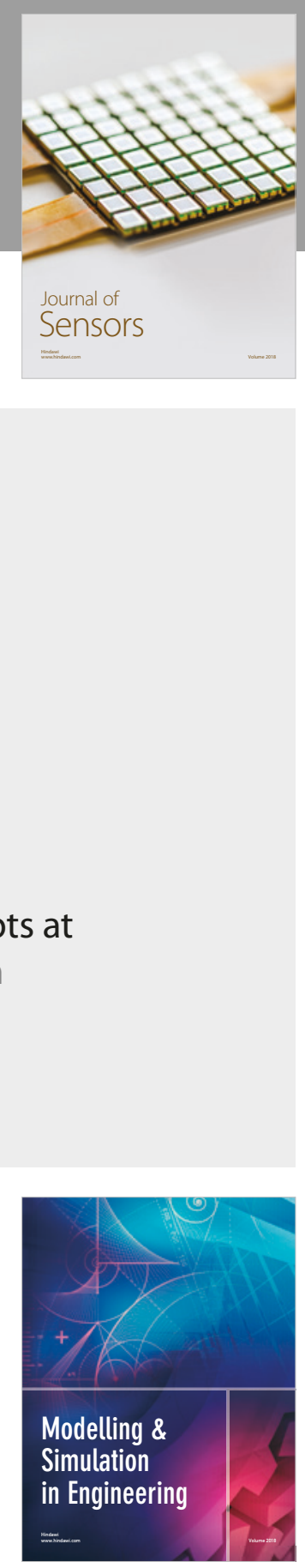

\section{Advances \\ Multimedia}
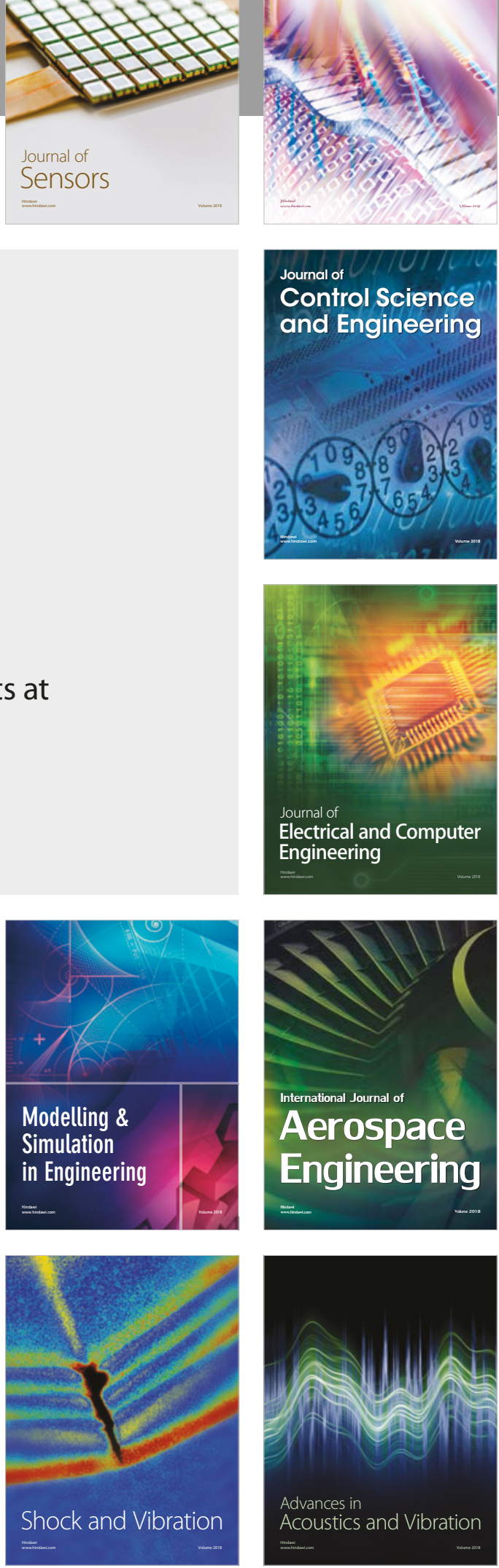